\title{
Temporal Floorplanning Using 3D-subTCG *
}

\author{
Ping-Hung Yuh ${ }^{1}$, Chia-Lin Yang ${ }^{1}$, Yao-Wen Chang ${ }^{2}$, Hsin-Lung Chen ${ }^{3}$ \\ ${ }^{1}$ Department of Computer Science and Information Engineering, National Taiwan University, Taipei, Taiwan \\ $\{$ r91089, yangc $\} @$ csie.ntu.edu.tw \\ ${ }^{2}$ Graduate Institute of Electronics Engineering \& Department of Electrical Engineering, National Taiwan University, Taipei, Taiwan \\ ywchang@cc.ee.ntu.edu.tw \\ Etron Technology Inc., Hsin-Chu, Taiwan \\ gis89536@cis.nctu.edu.tw
}

\begin{abstract}
Improving logic capacity by time-sharing, dynamically reconfigurable FPGAs are employed to handle designs of high complexity and functionality. In this paper, we use a novel topological floorplan representation, named $3 D$ subTCG (3-Dimensional sub-Transitive Closure Graph) to deal with the 3dimensional (temporal) floorplanning/placement problem, arising from dynamically reconfigurable FPGAs. The 3D-subTCG uses three transitive closure graphs to model the temporal and spatial relations between modules. We derive the feasibility conditions for the precedence constraints induced by the execution of the dynamically reconfigurable FPGAs. Because the geometric relationship is transparent to $3 \mathrm{D}$-subTCG and its induced operations, we can easily detect any violation of temporal precedence constraints on 3D-subTCG. We also derive important properties of the 3D-subTCG to reduce the solution space and shorten the running time for $3 \mathrm{D}$ (temporal) foorplanning/placement. Experimental results show that our 3D-subTCG based algorithm is very effective and efficient.
\end{abstract}

\section{Introduction}

A Field Programmable Gate Arrays (FPGA) is a (re)programmable logic device that implements multilevel logic. Traditionally, an FPGA needs to be reconfigured as a whole. Recently, several vendors have proposed architectures that allow partially dynamic reconfiguration, such as the Xilinx XC4000E FPGAs [18], the Atmel AT6000 Series FPGAs [2], the Xilinx XC6200 Series FPGAs [11], and Xilinx Virtex Series FPGAs [20].

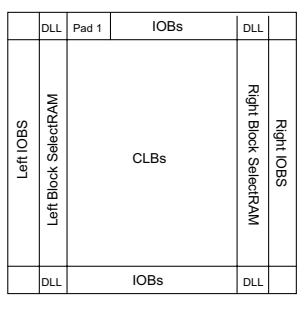

(a)

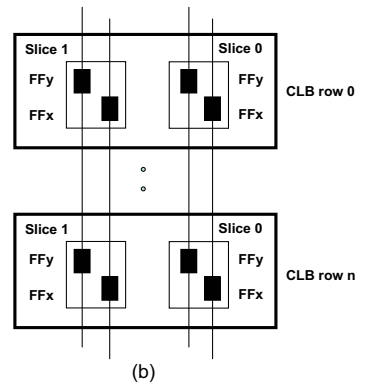

(b)
Figure 1: (a) The Virtex architecture. (b) One-column of a 2-slice Virtex CLB.

Figure 1(a) shows the Xilinx Virtex model [20]. The Virtex configuration memory can be considered as an array of bits. The bits of one-bit width that extend from the top to the bottom of the array constitute a vertical frame, which is the smallest portion of the configuration memory (i.e., the atomic unit that can be written to or read from in this device). Several frames are grouped together into larger units called columns. Figure 1(b) shows one column of configurable logic blocks (CLBs for short). In such a device, we have to specify a full column of a chip for reconfiguration and read-in/out of flip-flop of contents.

Because of the partial reconfiguration capability in an FPGA, studies have shown that an FPGA-based reconfigurable hardware system can improve performance for many applications [10]. A reconfigurable system is usually composed of a host processor and an FPGA coprocessor, called a reconfigurable functional unit (RFU) [3]. An RFU, which can be reconfigured during program execution, may have various configurations at different time. Figure 2(a) shows a program with four parts of codes mapped into RFU operations (called RFUOPS or modules). Because of the place constraint, we may not load all the modules into the device at the same time. Therefore,

\footnotetext{
*Yao-Wen Chang's work was partially supported by the National Science Council of Taiwan under Grant No. NSC 91-2215-E-002-038.
}
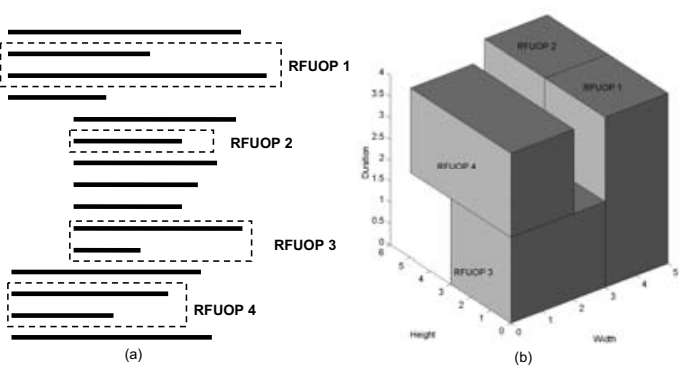

Figure 2: (a) A running program. (b) A 3D-placement of the running program.

how to place these modules into the RFU becomes a 3-D placement problem as shown in Figure 2(b). We may denote each module as a 3-D box with spatial dimensions $x$ and $y$ and the temporal dimension $t$. There exists temporal relation among scheduled modules since the result of one module may be needed by another one. The objective of temporal floorplanning is to allocate modules in the RFU to optimize the area and execution time without violating the temporal constraints.

\subsection{Previous Work}

Teich et al. in [17], first used component graphs to deal with such a problem assuming no dependence among scheduled modules. They derived necessary and sufficient conditions for a feasible placement and proposed an enumeration scheme by using a branch-and-bound tree search algorithm to find a feasible solution. In practice, however, there often exist temporal precedence constraints among scheduled modules since the output of one module may be needed as the input of another module. Therefore, Fekete et al. in [7] later extended their work to solve the placement problem with temporal precedence constraints by using an additional dependency graph. Bazargan et al. in their pioneering works [3], [4] and [5] considered both offline placement (3D template placement) and online placement. In the offline placement, they modeled each RFUOP as a 3D box and fixed the width and height of the RFU. They proposed a 3D-floorplanner which implements four effective methods, including one greedy method called KAMER-BF (Keep All Maximal Empty Rectangle with Best Fit). In the online placement, they allocated the free space of RFU to an RFUOP dynamically based on different greedy methods (e.g. best-fit and first-fit).

\subsection{Our Contribution}

In this paper, we solve the 3-dimensional floorplanning/placement problems of the general reconfigurable architecture by using a novel topological floorplan representation, called 3D-subTCG (3-Dimensional sub-Transitive Closure Graph). To the best knowledge of the authors, this is the first work that uses a topological representation to handle the 3-dimensional placement problem of a dynamically reconfigurable device.

Transitive closure graphs were previously proposed to handle classical 2D floorplanning/placement problems [15]. The main challenge to solve the $3 \mathrm{D}$ floorplanning problems is that there exists additional temporal precedence constraints, for which some tasks must be executed before other tasks start. We use the 3D-subTCG which consists of three transitive closure graphs to model the temporal as well as the spatial relations between tasks/modules. We derive the feasibility conditions for the temporal precedence and the spatial constraints induced by the execution of the dynamically reconfigurable FPGAs. Because the geometric relationship is transparent to the 3D-subTCG and its induced operations, we can easily detect any violation of temporal precedence and spatial constraints in the 3DsubTCG. Therefore, we can guarantee a feasible solution without resorting 
to time-consuming post-processing to remove infeasible ones. We also derive important properties of the 3D-subTCG to reduce the solution space and shorten the running time for 3D (temporal) foorplanning/placement. Experimental results show that our 3D-subTCG based algorithm can obtain significantly better floorplans than the Sequence Triplet (ST) representation (35.18\% deadspace in ST v.s. $14.86 \%$ in $3 \mathrm{D}$-subTCG). The running-time requirement of 3D-subTCG is also significantly smaller than ST (312.18 sec as ST v.s. $166.46 \mathrm{sec}$ as 3D-subTCG).

The remainder of this paper is organized as follows. Section 2 formulates the temporal floorplanning problem. Section 3 reviews the TCG representation and presents the 3D-subTCG for temporal floorplanning. Section 4 introduces our temporal floorplanning algorithm. Section 5 reports the experimental results. Finally, conclusions are given at Section 6.

\section{Formulation}

In the reconfigurable architecture, a task $v$ is loaded into the device for a period of time for execution. Let $V=\left\{v_{1}, v_{2}, \ldots, v_{m}\right\}$ be a set of $m$ tasks whose widths, heights, and durations are denoted by $W_{i}, H_{i}$, and $T_{i}, 1 \leq$ $i \leq m$. Let $\left(x_{i}, y_{i}\right)\left(\left(x_{i}^{\prime}, y_{i}^{\prime}\right)\right)$ denote the coordinate of the bottom-left (topright) corner of a task $v_{i}$ and , $1 \leq i \leq m$, on the chip. We use $t_{i}\left(t_{i}^{\prime}\right)$ to represrent the starting (ending) time of $v_{i}, 1 \leq i \leq m$, scheduled in the reconfigurable device.

To guarantee the correctness of the functions in the reconfigurable architecture, we must satisfy temporal precedence requirements, which describe the temporal ordering among tasks. We refer to the temporal precedence requirements as precedence constraints. Let $D=\left\{\left(v_{i}, v_{j}\right) \mid 1 \leq i, j \leq m, i \neq\right.$ $j\}$ denote the precedence constraints for the tasks $v_{i}$ and $v_{j}$. The precedence constraints should not be violated during floorplanning/placement.

In order to measure the quality of a floorplan, we consider the following objective functions:

- Volume (the minimum bounding box of a placement): In a temporal floorplanning, we need to consider the area of a device and the total execution time tradeoff. If we use a larger device, the total execution time could be shorten. In contrast, it takes longer time if a smaller one is used. Therefore, we shall minimize the product of the area of the device and the total execution time.

- Wirelength (the summation of half bounding box of interconnections): Due to the special architecture of the reconfigurable device, the method to estimate the wirelength in the temporal floorplanning is different from the traditional floorplanning/placement problem. Given a net, those nodes in the net may be executed at the same time or at different times. If they are executed at the same time, we can estimate the wirelength according to their geometric distance directly. However, we have to project all nodes into the same time frame before computing their wirelength in the other condition.

- Communication overhead: We quantify the communication overhead based on the Xilinx Virtex XCV1000 described in Section 1. Similar to the work by Fekete et al. [7], we assume that a task communicates with another task (data-dependence) in the following way: the results of a CLB, which are read by the successor task, are first written to external memory through a bus interface. The dependent task, which has been loaded at the specified position, then perform a read-in of the results. Recall that a frame is the atomic unit that can be written to or read from. Each frame contains 1248 bits and the bus width is only 8 bit. Thus, it takes approximately $1248 / 8+24=180$ clock cycles in each read-in or read-out, where the 24 cycles are the configuration overhead of the bus interface as described on the Xilinx FPGA data book [20]. Therefore, the communication overhead of each reconfiguration takes $360 \times f$ clock cycles time (we should first write the data to the external memory and then read back the data) if data in $f$ columns need to be transferred.

- Reconfiguration overhead: As described in Section 1, Xilinx Virtex XCV1000 is column-oriented (i.e., all bits in one column should be updated in each read-in or read-out). Suppose that a task $v_{i}$ occupies $W_{i} \times H_{i}$ CLBs. We have to reconfigure $H_{i}$ columns of CLBs in each reconfiguration. As an example, each CLB column in a Virtex FPGA consists of 48 frames, which takes $(1248 / 8) \times 48+24=7512$ clock cycles to configure per CLB column. This means we need $W_{i} \times 7512$ clock cycles in total if the addresses in the column are incrementally updated.

In this paper, we treat a task $v_{i}$ as a three-dimensional box. A placement $\mathcal{P}$ is an assignment of $\left(x_{i}, y_{i}, t_{i}\right)$ for each $v_{i}, 1 \leq i \leq m$, such that no two boxes overlap and all precedence constraints are satisfied. The goal of temporal floorplanning is to optimize a predefined cost metric (defined in the above)induced by a placement.

\section{3D-subTCG for Temporal Floorplanning}

\subsection{Review of TCG}

We first review the TCG representation presented in [15]. TCG uses two graphs, a horizontal transitive closure graph $C_{h}$ and a vertical transitive closure graph $C_{v}$, to describe the geometric relations among modules. For two non-overlap modules $b_{i}$ and $b_{j}, b_{i}$ is said to be horizontally (vertically) related to $b_{j}$, denoted by $b_{i} \vdash b_{j}\left(b_{i} \perp b_{j}\right)$, if $b_{i}$ is on the left (bottom) side of $b_{j}$ and their projections on $y(x)$ axis overlap. For two non-overlap modules $b_{i}$ and $b_{j}, b_{i}$ is said to be diagonally related to $b_{j}$ if $b_{i}$ is on the left side of $b_{j}$, and their projections on the $x$ axis and $y$ axis do not overlap. To simplify the operations on geometric relations, we treat a diagonal relation as a horizontal one, unless there exists a chain of vertical relations from $b_{i}$ $\left(b_{j}\right)$, followed by the modules enclosed with the rectangle defined by the two closest corners of $b_{i}$ and $b_{j}$, and finally to $b_{j}\left(b_{i}\right)$, for which we make $b_{i} \perp b_{j}\left(b_{j} \perp b_{i}\right)$. For each module $b_{i}$, we introduce one node $n_{i}$ both in $C_{h}$ and $C_{v}$. If $b_{i} \vdash b_{j}$, a directed edge $\left(n_{i}, n_{j}\right)$ is constructed in $C_{h}$. Similarly, we construct a directed edge $\left(n_{i}, n_{j}\right)$ in $C_{v}$ if $b_{i} \perp b_{j}$. Figure 3(a) shows a placement with five modules $a, b, c, d$, and $e$ whose widths and heights are $(2,1),(2,2),(3,2),(1,2)$, and $(3,1)$, respectively. Figure $3(\mathrm{~b})$ shows the TCG corresponding to the placement of Figure 3(a). The weight of each node in $C_{h}\left(C_{v}\right)$ represents the width (height) of the corresponding module $b_{i}$. Since $b_{a} \vdash b_{b}$, we construct a directed edge $\left(n_{a}, n_{b}\right)$ in $C_{h}$. Similarly, since $b_{a} \perp b_{c}$, a directed edge $\left(n_{a}, n_{c}\right)$ is constructed in $C_{v}$.

TCG has the following three feasibility properties [15]:

1. $C_{h}$ and $C_{v}$ are acyclic.

2. Each pair of nodes must be connected by exactly one edge either in $C_{h}$ or in $C_{v}$.

3. The transitive closure of $C_{h}\left(C_{v}\right)$ is equal to $C_{h}\left(C_{v}\right)$ itself. $^{1}$

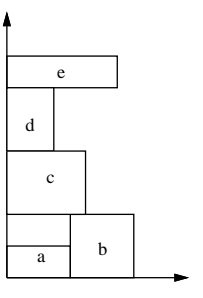

(a)

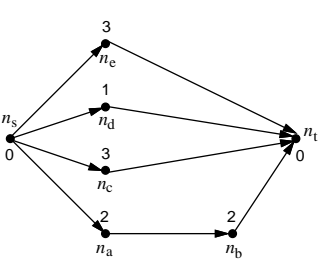

Augmented $C_{h}$
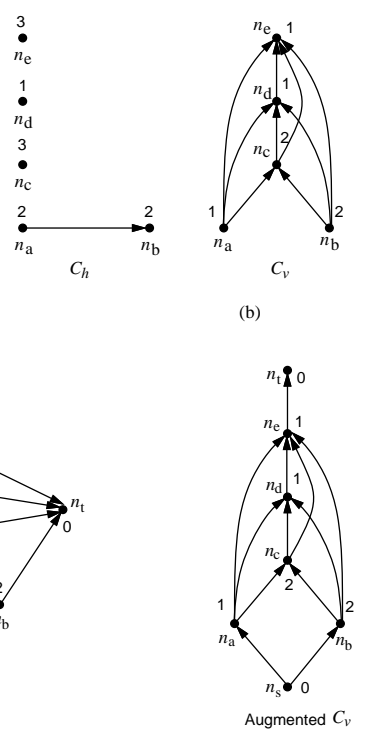

Figure 3: (a) A placement. (b) TCG. (c) Augmented TCG (augmented $C_{h}$ and $C_{v}$ ).

The first property ensures that a module $b_{i}$ cannot be both left and right to (below and above) another module $b_{j}$ in a placement. The second property guarantees that no two modules overlap since each pair of modules have exactly one of the horizontal or vertical relation. The third property eliminates redundant solutions. Figure 4 illustrates the third property. As shown in Figure 4 (a), since there is a path from node $n_{c}$ to node $n_{e}$ in $C_{v}$, the edge $\left(n_{c}, n_{e}\right)$ must be in $C_{v}$. If we place the edge $\left(n_{c}, n_{e}\right)$ into $C_{h}$, as shown in Figure 4 (c), the resulting area of placement must be larger or equal to the configuration of Figure 4 (a). Figure 4 (b) and (d) shows the two placement. The third property eliminates this redundant solution.

Given a TCG, a placement can be obtained in $O\left(\mathrm{~m}^{2}\right)$ time by performing a well-known longest path algorithm [14] on TCG, where $m$ is the number of modules. To facilitate the implementation of the longest path algorithm, the two closure graphs can be augmented as follows. For each closure graph, we introduce two special nodes with zero wights, the source $n_{s}$ and the sink $n_{t}$, and construct an edge from $n_{s}$ to each node with in-degree equal to zero and also from each node with out-degree equal to zero to $n_{t}$. Figure 3(c) shows the augmented TCG for the TCG shown in Figure 3(b).

\footnotetext{
${ }^{1}$ The transitive closure of a directed acyclic graph $G$ is defined as the graph $G^{\prime}=$ $\left(V, E^{\prime}\right)$, where $E^{\prime}=\left\{\left(n_{i}, n_{j}\right)\right.$ : there is a path from node $n_{i}$ to node $n_{j}$ in $\left.G\right\}$
} 


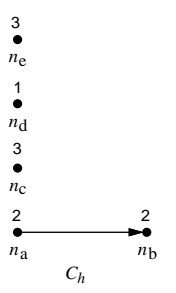

(a)

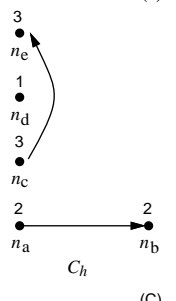

(C)

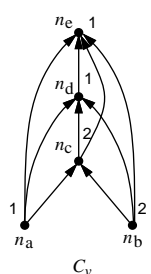

$C_{v}$

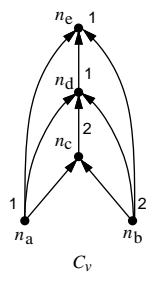

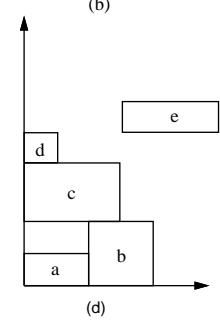

Figure 4: (a) A feasible TCG that the edge $\left(n_{c}, n_{e}\right)$ lies in $C_{v}$. (b) The corresponding placement of Figure 4 (a). (c) A non-feasible TCG that the edge $\left(n_{c}, n_{e}\right)$ lies in $C_{h}$. (d) The corresponding placement of Figure 4 (c).

Let $L_{h}\left(n_{i}\right)\left(L_{v}\left(n_{i}\right)\right)$ denote the weight of the longest path from $n_{s}$ to $n_{i}$ in the augmented $C_{h}\left(C_{v}\right) . L_{h}\left(n_{i}\right)\left(L_{v}\left(n_{i}\right)\right)$ can be determined by performing the single source longest path algorithm on the augmented $C_{h}\left(C_{v}\right)$ in $O\left(\mathrm{~m}^{2}\right)$ time, where $m$ is number of modules. The coordinate $\left(X_{i}, Y_{i}\right)$ of a module $b_{i}$ is given by $\left(L_{h}\left(n_{i}\right), L_{v}\left(n_{i}\right)\right)$. Further, the coordinates of all modules are determined in the topological order in $C_{h}\left(C_{v}\right)$. Since the respective width and height of the placement for the given TCG are $L_{h}\left(n_{t}\right)$ and $L_{v}\left(n_{t}\right)$, the area of the placement is given by $L_{h}\left(n_{t}\right) \times L_{v}\left(n_{t}\right)$. Since each module has a unique coordinate after packing, there exists a unique TCG corresponding to any placement.

\subsection{D-subTCG}

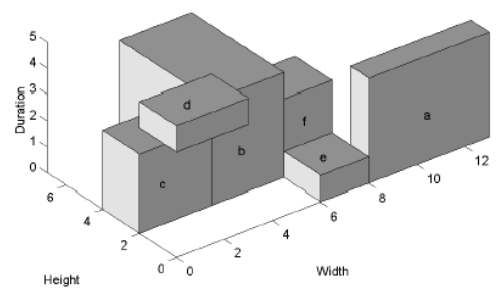

Figure 5: A placement.
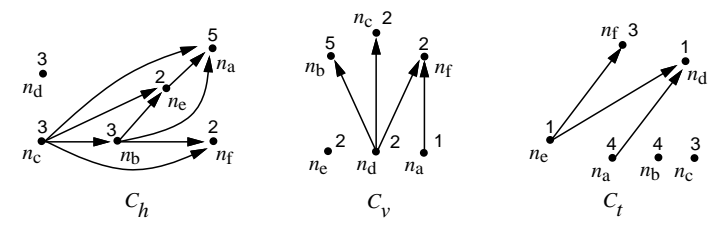

Figure 6: The corresponding 3D-subTCG of Figure 5.

As shown in the previous section, TCG describes the geometric relations among modules based on two graphs, $C_{h}$ and $C_{v}$. For a dynamically reconfigurable device, there exists temporal ordering among tasks. For two tasks $v_{i}$ and $v_{j}, v_{i}$ is said to be temporally related to $v_{j}$, denoted by $v_{i} \prec v_{j}$, if $v_{i}$ must be executed before $v_{j}$ starts. To solve the 3D floorplanning/placement problems, we need to consider the temporal and spatial relations at the same time. Therefore, we introduce a new graph to model the temporal relations among tasks, namely a temporal transitive closure graph $C_{t}$. This new representation is called 3D-subTCG, which contains three transitive graphs, $C_{h}$, $C_{v}$ and $C_{t}$. For each task $v_{i}$, we construct one node $n_{i}$ in each graph. If $v_{i} \vdash v_{j}\left(v_{i} \perp n_{j}\right)$, we construct one edge $\left(n_{i}, n_{j}\right)$ in $C_{h}\left(C_{v}\right)$. If $v_{i}$ must be executed before $v_{j}$, we construct an edge $\left(n_{i}, n_{j}\right)$ in $C_{t}$.

Figure 5 shows a placement with six tasks $a, b, c, d, e$, and $f$ whose widths, heights and durations are $(5,1,4),(3,5,4),(3,2,3),(3,2,1),(2,2$, $1)$, and $(2,2,3)$, respectively. Figure 6 shows the 3 D-subTCG corresponding to the placement of Figure 5. The value associated with a node in $C_{h}\left(C_{v}\right.$ or $C_{t}$ ) gives the width (height or duration) of the corresponding task, and the edge $\left(n_{i}, n_{j}\right)$ in $C_{h}\left(C_{v}\right.$ or $\left.C_{t}\right)$ denotes the horizontal (vertical or temporal) relation of $v_{i}$ and $v_{j}$. In Figure 6 , since task $v_{c}\left(v_{a}\right)$ is left to (below) $v_{b}$ $\left(v_{f}\right)$, there exists an edge $\left(n_{c}, n_{b}\right)\left(\left(n_{a}, n_{f}\right)\right)$ in $C_{h}\left(C_{v}\right)$. Similarly, since task $v_{a}$ must be executed before task $v_{d}$, there exists an edge $\left(n_{a}, n_{d}\right)$ in $C_{t}$. To obtain the coordinate of each task, we apply the longest path algorithm to the three graphs in a 3D-subTCG. (See Section 3.1 for the details.)

3D-subTCG has the following three feasibility properties:

1. $C_{h}, C_{v}$ and $C_{t}$ are acyclic.

2. Each pair of nodes must have exactly one edge either in $C_{h}, C_{v}$ or $C_{t}$.

3. There must exist an edge $\left(n_{i}, n_{j}\right)$ if there is a path from $n_{i}$ to $n_{j}$ in one graph and there exists no closure edge between $n_{i}$ and $n_{j}$ in other graphs.

The first two properties, which are the same as TCG, guarantee that a solution is feasible. The third property is to eliminate the redundant solutions. An edge $\left(n_{i}, n_{j}\right)$ is said to be a closure edge if there exists a path from node $n_{i}$ to node $n_{j}$ except the edge $\left(n_{i}, n_{j}\right)$ itself. For example, the edges $\left(n_{b}, n_{a}\right),\left(n_{c}, n_{a}\right),\left(n_{c}, n_{e}\right)$, and $\left(n_{c}, n_{f}\right)$ in $C_{h}$ of Figure 6 are closure edges. If there exists a path from node $n_{i}$ to node $n_{j}$ in one graph, the closure edge $\left(n_{i}, n_{j}\right)$ should appear in the same graph instead of others to eliminate the redundant solutions as explained in section 3.1. However, before adding a new closure edge $\left(n_{i}, n_{j}\right)$ after each operation, we need to make sure that there exists no closure edges between $n_{i}$ and $n_{j}$ in other graphs. Figure 7 illustrates this scenario. Figure 7 (a) shows a 3D-subTCG that node $n_{a}$ and $n_{b}$ have a closure edge in $C_{v}$. Figure 7 (b) shows the resulting graph after deleting edge $\left(n_{e}, n_{b}\right)$ in $C_{t}$ and adding edge $\left(n_{b}, n_{e}\right)$ to $C_{h}$. Now there is a path from $n_{b}$ to $n_{a}$ in $C_{h}$. However, in order to maintain the second property, we cannot add the closure edge $\left(n_{b}, n_{a}\right)$ in $C_{h}$ since $\left(n_{a}, n_{b}\right)$ has already existed in $C_{v}$.
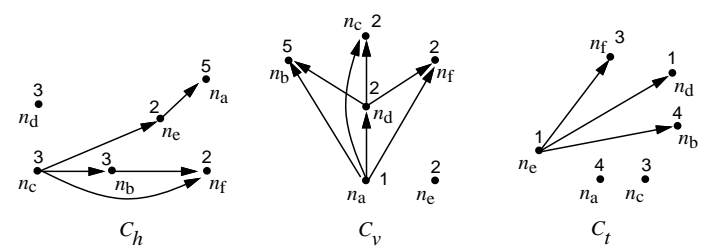

(b)
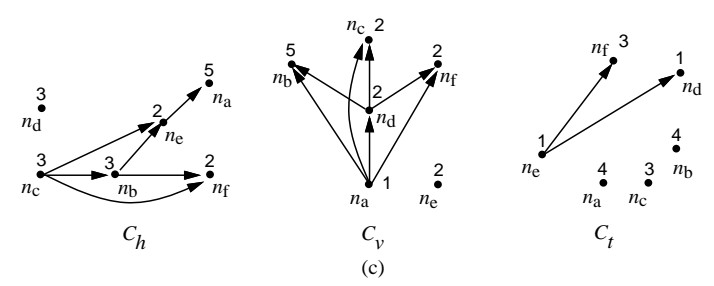

Figure 7: (a) A 3D-subTCG with only one path between node $n_{a}$ and $n_{b}$ in $C_{v}$. (b) A 3D-subTCG contains two paths in $C_{h}$ and $C_{v}$ between node $n_{a}$ and $n_{b}$.

\section{Temporal Floorplanning Algorithm}

Our algorithm is based on simulated annealing [12]. Given an initial 3DsubTCG, we perturb the 3D-subTCG to obtain a new 3D-subTCG. The cost function $\Phi$ used in our algorithm is given by

$$
\Phi=\alpha V+\beta W+\gamma O,
$$

where $V$ is the volume of the placement, $W$ is the total wirelength, $O$ is the reconfiguration and communication overheads, and $\alpha, \beta$, and $\gamma$ are userspecified constants. In this section, we first describe how to identify a reduction edge, and then show the perturbation operations in simulated annealing. Finally, we introduce the feasibility condition that a 3D-subTCG must satisfy during each perturbation in order to maintain the correct temporal ordering among tasks.

\subsection{Reduction Edge Identification}

First we illustrate the concept of reduction edge. An edge $\left(n_{i}, n_{j}\right)$ is called reduction edge if there does not exist another path from node $n_{i}$ to node $n_{j}$ except the edge $\left(n_{i}, n_{j}\right)$ itself. For example, the edges $\left(n_{b}, n_{f}\right),\left(n_{b}, n_{e}\right)$ and $\left(n_{e}, n_{a}\right)$ in $C_{h}$ of Figure 6 are reduction edges. Recall that 3D-subTCG is formed by directed acyclic transitive closure graphs. Given an arbitrary node $n_{i}$ in one transitive closure graph, there exists at least one reduction edge $\left(n_{i}, n_{j}\right)$, where $n_{j} \in F_{\text {out }}\left(n_{i}\right)$. Here we define the fan-in (fan-out) of a node $n_{i}$, denoted by $F_{\text {in }}\left(n_{i}\right)\left(F_{\text {out }}\left(n_{i}\right)\right)$, as the nodes $n_{j}$ 's with edges $\left(n_{j}, n_{i}\right)\left(\left(n_{i}, n_{j}\right)\right)$. For nodes $n_{k}, n_{l} \in F_{\text {out }}\left(n_{i}\right)$, the edge $\left(n_{i}, n_{k}\right)$ cannot be a reduction edge if $n_{k} \in F_{\text {out }}\left(n_{l}\right)$. Hence, we remove those nodes in $F_{\text {out }}\left(n_{i}\right)$ that are fan-outs of others. The edges between $n_{i}$ and the remaining nodes in $F_{\text {out }}\left(n_{i}\right)$ are reduction edges. In the $C_{h}$ of Figure 6, 
$F_{\text {out }}\left(n_{c}\right)=\left\{n_{a}, n_{b}, n_{e}, n_{f}\right\}$. Since $n_{a}, n_{e}$, and $n_{f}$ belong to $F_{\text {out }}\left(n_{b}\right)$, edges $\left(n_{c}, n_{a}\right)$ and $\left(n_{c}, n_{f}\right)$ are closure edges while $\left(n_{c}, n_{b}\right)$ is a reduction one. The reason for identifying reduction edges is that the operations defined below are only applied to reduction edges. The time complexity of finding such a reduction edge is $O\left(\mathrm{~m}^{2}\right)$, where $m$ is the number of modules (tasks) [15].

\subsection{Solution Perturbation}

We define the following five operations to perturb a 3D-subTCG:

- Rotation: Rotate a task.

- Swap: Swap two nodes in $C_{h}, C_{v}$, and $C_{t}$.

- Reverse: Reverse a reduction edge in $C_{h}, C_{v}$, or $C_{t}$.

- Move: Move a reduction edge from one graph $\left(C_{h}, C_{v}\right.$, or $\left.C_{t}\right)$ to another graph.

- Transpositional Move: Move a reduction edge from one graph $\left(C_{h}\right.$, $C_{v}$, or $C_{t}$ ) to another graph, and then transpose the two nodes associated with the edge. It is clear later that this operation is different from performing Move followed by Reverse.

Note that Rotation, Swap, Reverse, and Move are first introduced in [15], which can be performed in respective $O(1), O(1), O\left(\mathrm{~m}^{2}\right)$, and $O\left(\mathrm{~m}^{2}\right)$ times, where $m$ is the number of modules (tasks). Further, the resulting graph after performing any of these operations on a 3D-subTCG is still a 3D-subTCG. Rotation and Swap do not change the topology of 3D-subTCG, while Revere, Move, and Transpositional Move do. Therefore, to maintain the properties of a 3D-subTCG, we may need to update the resulting graphs after performing Reverse, Move and Transpositional Move. Further, in order to guarantee that the precedence constraints are not violated by these operations, we shall perform feasibility detection, which are described in section 4.3. We first detail the operations in the following.

\subsubsection{Rotation}

To rotate a task $v_{i}$, we only need to exchange the weights of the corresponding nodes $n_{i}$ in $C_{h}, C_{v}$, and $C_{t}$. Figure 8 (b) shows the result after rotating the module a in Figure 8.

\subsubsection{Move}

The Move operation moves a reduction edge $\left(n_{i}, n_{j}\right)$ in one graph to one of the others in a 3D-subTCG. Move could switch the relations of the two tasks $v_{i}$ and $v_{j}$ between a horizontal relation and a vertical one. For two tasks $v_{i}$ and $v_{j}, v_{i} \vdash v_{j}\left(v_{i} \perp v_{j}\right)$ if there exists a reduction edge $\left(n_{i}, n_{j}\right)$ in $C_{h}$ $\left(C_{v}\right)$; after moving the edge $\left(n_{i}, n_{j}\right)$ to $C_{v}\left(C_{h}\right)$, we have the new geometric relation $v_{i} \perp v_{j}\left(v_{i} \vdash v_{j}\right)$. Move could also change the temporal relation of the two tasks $v_{i}$ and $v_{j}$. For two tasks $v_{i}$ and $v_{j}, v_{i} \prec v_{j}$ if there exists a reduction edge $\left(n_{i}, n_{j}\right)$ in $C_{t}$; after moving the edge $\left(n_{i}, n_{j}\right)$ to $C_{h}\left(C_{v}\right)$, we change the temporal relation into the new geometric relation $v_{i} \vdash v_{j}$ $\left(v_{i} \perp v_{j}\right)$. If there exists a reduction edge $\left(n_{i}, n_{j}\right)$ in $C_{h}\left(C_{v}\right)$; after moving the edge $\left(n_{i}, n_{j}\right)$ to $C_{t}$, we have the new temporal relation $v_{i} \prec v_{j}$.

To move a reduction edge $\left(n_{i}, n_{j}\right)$ from one graph $G$ to another graph $G^{\prime}$, we first delete the edge $\left(n_{i}, n_{j}\right)$ from $G$ and then add $\left(n_{i}, n_{j}\right)$ to $G^{\prime}$. For each node $n_{k} \in F_{\text {in }}\left(n_{i}\right) \cup\left\{n_{i}\right\}$ and $n_{l} \in F_{\text {out }}\left(n_{j}\right) \cup\left\{n_{j}\right\}$, we shall check whether the edge $\left(n_{k}, n_{l}\right)$ exists in $G^{\prime}$. If $G^{\prime}$ contains the edge, we do nothing; otherwise, we need to add the edge to $G^{\prime}$ and delete the corresponding edge $\left(n_{k}, n_{l}\right)$ or $\left(n_{l}, n_{k}\right)$ in $G$ or $G^{\prime \prime}$, if any, to maintain the properties of the 3D-subTCG. Figure 8 (c) shows the result of moving the edge $\left(n_{c}, n_{b}\right)$ in $C_{h}$ of Figure 8 (b) to $C_{t}$.

\subsubsection{Swap}

To swap nodes $n_{i}$ and $n_{j}$ of two tasks $v_{i}$ and $v_{j}$, we only need to exchange the nodes $n_{i}$ and $n_{j}$ in $C_{h}, C_{v}$, and $C_{t}$. Figure 9 (a) shows the result of swapping nodes $n_{b}$ and $n_{d}$ shown in Figure 8 (c).

\subsubsection{Reverse}

The Reverse operation reverses the direction of a reduction edge $\left(n_{i}, n_{j}\right)$ in one graph. For two modules $v_{i}$ and $v_{j}, v_{i} \vdash v_{j}\left(v_{i} \perp v_{j}\right)$ if there exists a reduction edge $\left(n_{i}, n_{j}\right)$ in $C_{h}\left(C_{v}\right)$; after reversing the edge $\left(n_{i}, n_{j}\right)$, we have the new geometric relation $v_{j} \vdash v_{i}\left(v_{j} \perp v_{i}\right)$. Similarly, $v_{i} \prec v_{j}$ if there exists a reduction edge $\left(n_{i}, n_{j}\right)$ in $C_{t}$; after reversing the edge $\left(n_{i}, n_{j}\right)$, we have the new temporal relation $v_{j} \prec v_{i}$.

To reverse a reduction edge $\left(n_{i}, n_{j}\right)$ in a graph, we first delete the edge from the graph, and then add the edge $\left(n_{j}, n_{i}\right)$ to the same graph. Similar to the Move operation, for each node $n_{k} \in F_{i n}\left(n_{j}\right) \cup\left\{n_{j}\right\}$ and $n_{l} \in F_{\text {out }}\left(n_{i}\right) \cup\left\{n_{i}\right\}$ in the new graph, we shall check whether the edge $\left(n_{k}, n_{l}\right)$ exists in the new graph. If the graph contains the edge, we do nothing; otherwise, we need to add the edge to the graph and delete the corresponding edge $\left(n_{k}, n_{l}\right)$ or $\left(n_{l}, n_{k}\right)$ in the other transitive closure graphs, if any, to maintain the properties of the 3D-subTCG. Figure 9 (b) shows the result after reversing the edge $\left(n_{e}, n_{a}\right)$ in $C_{h}$ of Figure 9 (a).

\subsubsection{Transpositional Move}

The Transpositional Move operation removes a reduction edge $\left(n_{i}, n_{j}\right)$ from one graph, and add an edge $\left(n_{j}, n_{i}\right)$ to one of the others in a 3D-subTCG.

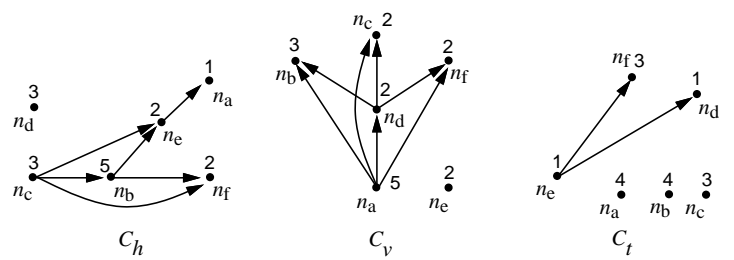

(a) Initial configuration of 3D-subsTCG

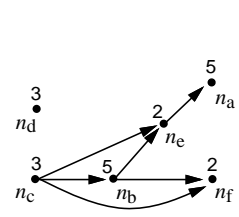

$C_{h}$

(b) Rotate $n_{\mathrm{a}}$

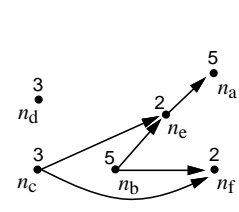

$C_{h}$

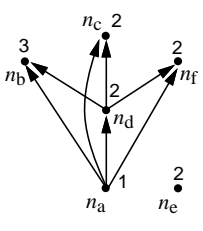

$C_{v}$

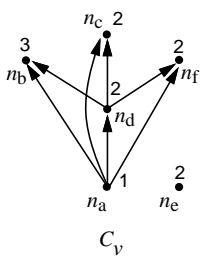

(c) Move $\left(n_{\mathrm{c}}, n_{\mathrm{b}}\right)$

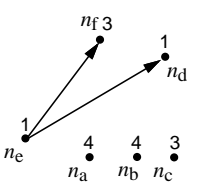

$C_{t}$

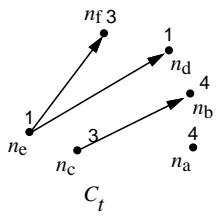

Figure 8: Examples of perturbations. (a) The initial 3D-subTCG $\left(C_{h}, C_{v}\right.$, and $\left.C_{t}\right)$ (b) The resulting 3D-subTCG after rotating the task $n_{a}$ shown in(a). (c) The resulting 3D-subTCG after moving the reduction edge $\left(n_{c}, n_{b}\right)$ from the $C_{h}$ of (b) to $C_{t}$.

In one case, Transpositional Move switches the geometric relation of the two tasks $v_{i}$ and $v_{j}$ between a horizontal relation and a vertical one and changes the ordering of the two tasks $v_{i}$ and $v_{j}$ in their geometric relation. For two tasks $v_{i}$ and $v_{j}, v_{i} \vdash v_{j}\left(v_{i} \perp v_{j}\right)$ if there exists a reduction edge $\left(n_{i}, n_{j}\right)$ in $C_{h}\left(C_{v}\right)$; after transpositionally moving the edge $\left(n_{i}, n_{j}\right)$ to $C_{v}$ $\left(C_{h}\right)$, we have the new geometric relation $v_{j} \perp v_{i}\left(v_{j} \vdash v_{i}\right)$. In the other case, Transpositional Move changes the temporal relation of the two tasks $v_{i}$ and $v_{j}$. For two tasks $v_{i}$ and $v_{j}, v_{i} \prec v_{j}$ if there exists a reduction edge $\left(n_{i}, n_{j}\right)$ in $C_{t}$; after transpositionally moving the edge $\left(n_{i}, n_{j}\right)$ to $C_{h}$ $\left(C_{v}\right)$, we change the temporal relation into the new geometric relation $v_{j} \vdash$ $v_{i}\left(v_{j} \perp v_{i}\right)$. If there exists a reduction edge $\left(n_{i}, n_{j}\right)$ in $C_{h}\left(C_{v}\right)$; after transpositionally moving the edge $\left(n_{i}, n_{j}\right)$ to $C_{t}$, we have the new temporal relation $v_{j} \prec v_{i}$

To transpositionally move a reduction edge $\left(n_{i}, n_{j}\right)$ from one graph $G$ to another graph $G^{\prime}$, we first delete the edge $\left(n_{i}, n_{j}\right)$ from $G$ and add $\left(n_{j}, n_{i}\right)$ to $G^{\prime}$. Similar to the Move operation, for each node $n_{k} \in F_{i n}\left(n_{j}\right) \cup\left\{n_{j}\right\}$ and $n_{l} \in F_{\text {out }}\left(n_{i}\right) \cup\left\{n_{i}\right\}$, we shall check whether the edge $\left(n_{k}, n_{l}\right)$ exists in $G^{\prime}$. If $G^{\prime}$ contains the edge, we do nothing; otherwise, we need to add the edge to $G^{\prime}$ and delete the corresponding edge $\left(n_{k}, n_{l}\right)$ or $\left(n_{l}, n_{k}\right)$ in $G$ or $G^{\prime \prime}$, if any, to maintain the properties of the 3D-subTCG. Figure 9 (c) shows the result of transpositionally moving the edge $\left(n_{e}, n_{b}\right)$ from $C_{t}$ of Figure 9 (b) to $C_{v}$. Note we delete the edge $\left(n_{a}, n_{e}\right)$ in $C_{h}$ and add it to $C_{v}$.

\subsection{Feasibility Detection}

To maintain the temporal ordering among tasks, the 3D-subTCG must guarantee that all precedence constraints are satisfied. Among the five operations mentioned above, Move, Swap, Reverse, and Transpositional Move could violate the constraints. We now show how to detect a violation during perturbation.

When we move an edge $\left(n_{i}, n_{j}\right)$ or reverse/transpositionally move $\left(n_{j}, n_{i}\right)$, the precedence constraint will be violated if $n_{l} \in F_{i n}\left(n_{i}\right) \cup\left\{n_{i}\right\}$, $n_{k} \in F_{\text {out }}\left(n_{j}\right) \cup\left\{n_{j}\right\}$, and $\left(n_{l}, n_{k}\right) \notin C_{t}$ since $\left(n_{l}, n_{k}\right) \in D$. As mentioned in Section 2, $D$ denotes the precedence constraints. When we swap two nodes $n_{i}$ and $n_{j}$, three scenarios could happen:

1. there exists a precedence constraint between $n_{i}$ and $n_{j}$,

2. neither of $n_{i}$ and $n_{j}$ has a precedence constraint, or

3. either $n_{i}$ or $n_{j}$ has precedence constraint.

In the first case, it is clear that we cannot swap the two nodes. However, if neither of $n_{i}$ and $n_{j}$ has a precedence constraint, we can swap $n_{i}$ and $n_{j}$ directly. Without loss of generality, we could assume that node $n_{i}$ has precedence constraints to detail the third case. If node $n_{j}$ has precedence constraints, we can apply the same approach to check the feasibility. In the first condition, $n_{i}$ has a precedence-constrained edge $\left(n_{i}, n_{k}\right)$, we can swap $n_{i}$ and $n_{j}$ without any violation if $n_{k} \in F_{\text {out }}\left(n_{j}\right)$ in $C_{t}$. On the other hand, if $n_{i}$ has a precedence-constrained edge $\left(n_{k}, n_{i}\right)$ and $n_{k} \in F_{i n}\left(n_{j}\right)$ in $C_{t}$, we can also swap $n_{i}$ and $n_{j}$. 


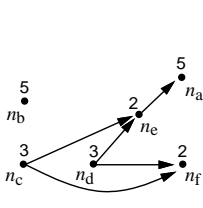

$C_{h}$

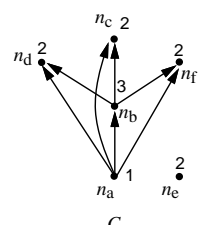

$C_{v}$

(a) Swap $\left(n_{\mathrm{b}}, n_{\mathrm{d}}\right)$

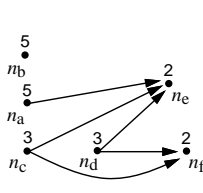

$C_{h}$

(b) Reverse $\left(n_{\mathrm{e}}, n_{\mathrm{a}}\right)$

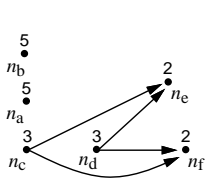

$C_{h}$
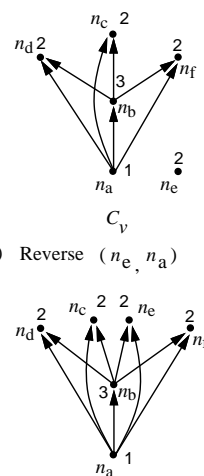

$C_{v}$
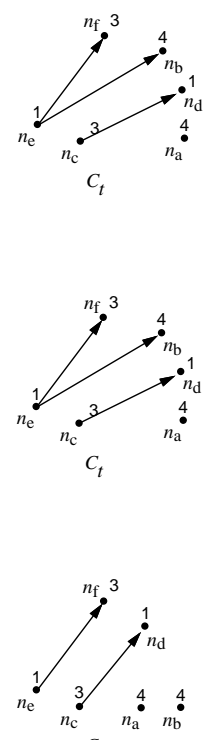

$C_{t}$

(c) Transpositional Move the edge $\left(n_{\mathrm{e}}, n_{\mathrm{b}}\right)$ from $C_{t}$ to $C_{v}$

Figure 9: Examples of perturbations (continued from Figure 8). (a) The resulting 3D-subTCG after swapping the nodes $n_{b}$ and $n_{d}$ shown in Figure 8(c). (b) The resulting 3D-subTCG after reversing the reduction edge $\left(n_{e}, n_{a}\right)$ in the $C_{h}$ shown in (a). (c) The resulting 3D-subTCG after transpositional moving the reduction edge $\left(n_{e}, n_{b}\right)$ from the $C_{t}$ of (b) to $C_{v}$.

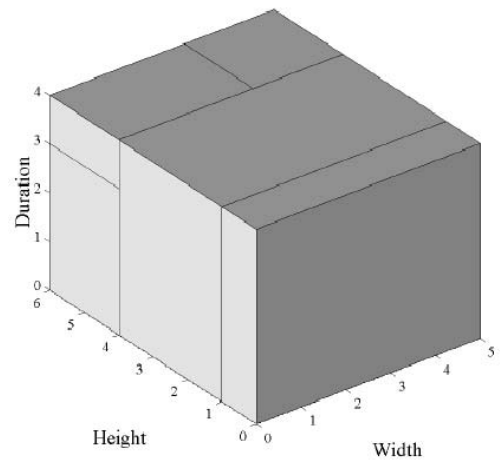

Figure 10: The resulting placement of 3D-subTCG in Figure 9(c).

Figure 11(a) shows the resulting $C_{h}, C_{v}$, and $C_{t}$ after swapping the nodes $n_{d}$ and $n_{e}$ in Figure 9(c). Assume that there exists a precedence-constrained edge $\left(n_{e}, n_{f}\right)$. The precedence constraint will be violated if we swap the two nodes $n_{d}$ and $n_{e}$ since $n_{f} \notin F_{\text {out }}\left(n_{d}\right)$ in the $C_{t}$. Figure 11(b) shows $C_{h}$, $C_{v}$, and $C_{t}$ after reversing the edge $\left(n_{d}, n_{e}\right)$ in the $C_{h}$ in Figure 9(c). Since $\left\{n_{e}\right\} \cap F_{i n}\left(n_{e}\right)=\left\{n_{c}, n_{e}\right\}$ and $\left\{n_{d}\right\} \cap F_{\text {out }}\left(n_{d}\right)=\left\{n_{d}, n_{f}\right\}$ in $C_{h}$, we shall check $\left(n_{e}, n_{f}\right)$ for the precedence constraint. If there exists a precedenceconstrained edge $\left(n_{e}, n_{f}\right)$, the precedence constraint will be violated.

By doing the feasibility detection during the Move, Reverse, Transpositional Move, or Swap operations, we can guarantee that the resulting 3DsubTCG still satisfies all the precedence constraints. We thus have the following theorem.

Theorem 1 The precedence constraints of a 3D-TCG are not violated by the Move, Swap, Reverse, or Transpositional Move operation with the feasibility detection.

\section{Experimental Results}

\begin{tabular}{|c||c||c||c||c||c||}
\hline \hline Circuit & $\begin{array}{c}\text { \# of } \\
\text { tasks }\end{array}$ & $\begin{array}{c}\text { Sum of } \\
\text { volume }\end{array}$ & Volume & $\begin{array}{c}\text { Dead space } \\
(\%)\end{array}$ & $\begin{array}{c}\text { time } \\
\text { (Sec.) }\end{array}$ \\
\hline \hline Circuit 1 & 10 & 512 & 512 & 0.0 & 8.3 \\
Circuit 2 & 10 & 480 & 480 & 0.0 & 1.9 \\
Circuit 3 & 10 & 1000 & 1000 & 0.0 & 9.7 \\
Circuit 4 & 20 & 3840 & 4032 & 4.7 & 25.2 \\
Circuit 5 & 30 & 4096 & 4608 & 11.1 & 127.8 \\
\hline
\end{tabular}

Table 1: Results of volume optimation (volume $=\mathrm{mm}^{2} \mathrm{x}$ clock cycles).
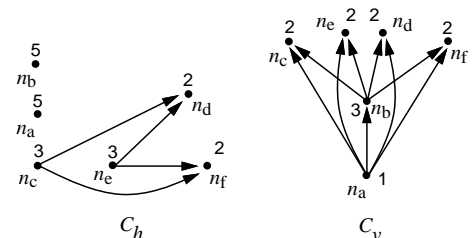

(a) Swap $\left(n_{\mathrm{d}}, n_{\mathrm{e}}\right)$
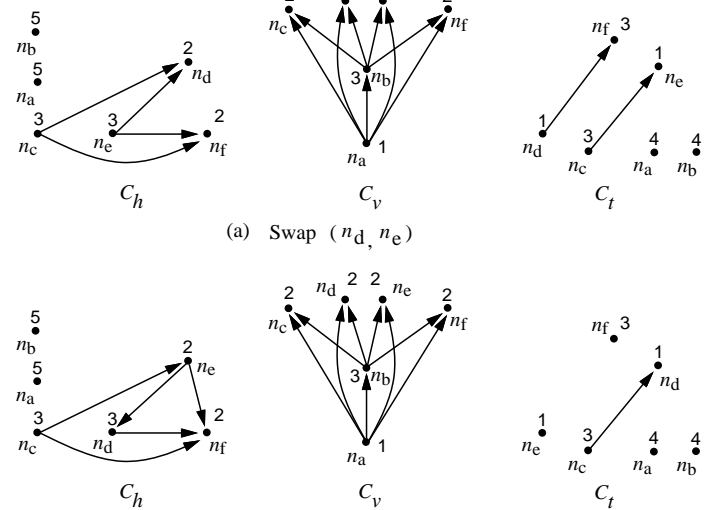

(b) Reverse the edge $\left(n_{\mathrm{d}}, n_{\mathrm{e}}\right)$ in $C_{h}$

Figure 11: (a) The resulting 3D-subTCG after swapping the nodes $n_{d}$ and $n_{e}$ shown in Figure 9(c). (b) The resulting 3D-subTCG after reversing the reduction edge $\left(n_{d}, n_{e}\right)$ in the $C_{h}$ shown in Figure 9(c).

Based on simulated annealing [12], we implemented the temporal floorplanning algorithm in the $\mathrm{C}++$ programming language on a $433 \mathrm{MHz}$ SUN Ultra-60 workstation with 1 GB memory. We compared 3D-subTCG with Sequence Triplet (ST). ST is extended from the well-known Sequence Pair (SP) [16], which is very popular for handling floorplanning/placement in both industry and academia ${ }^{2}$ A sequence triplet consists of three module sequences $\left(\Gamma_{x}, \Gamma_{y}, \Gamma_{z}\right)$. The relation between two modules is defined as follows: (1) if the sequence of two modules $a, b$ is the same (from left to right $)$ in $\left(\Gamma_{x}, \Gamma_{z}\right)$, i.e., $\left(\Gamma_{x}, \Gamma_{y}, \Gamma_{z}\right)=(.$. a..b..., ...., ..a..b...), it means that module $a$ is on the $Z^{+}$direction of module $b$; (2) if the sequence of the two modules $a, b$ is not the same in $\left(\Gamma_{x}, \Gamma_{z}\right)$, the $\left(\Gamma_{x}, \Gamma_{y}, \Gamma_{z}\right)$ is identical to Sequence Pair $\left(\Gamma_{x}, \Gamma_{y}\right)$. For example, the ST representation of Figure 5 is (dcbfea, cbeafd, dafebc). Based on the same simulated annealing scheme as that for 3D-subTCG, ST employs the following three perturbation operations: (1) M1: randomly swap two modules in one of the $\Gamma_{X}, \Gamma_{y}$, and $\Gamma_{z}$ sequences; (2) M2: randomly swap two modules in $\Gamma_{X}, \Gamma_{y}$, and $\Gamma_{z}$ simultaneously; (3) M3: randomly choose one module and change its height with width, width with length, or length with height (i.e., 3D rotation). We implemented the ST algorithm with the same simulated annealing engine as that of 3D-subTCG with the limiting that rotation can only change width and height (i.e., duration remains the same), and added precedence constraints, reconfiguration overheads and communication overheads for comparative studies.

To verify our algorithm, we first tested 3D-subTCG on five synthetic circuits that can be packed without deadspace. Table 1 shows the results. Note that the volume of a placement is the minimum bounding box enclosing the placement. We can see that 3D-subTCG obtains the optimal placements for the first three test cases and near optimal solutions for the last two larger circuits, all in reasonable time. The results show that our approach is very effective for cost optimization.

\begin{tabular}{|c||c||c||c||c||c|}
\hline \hline Circuit & $\begin{array}{c}\text { \# of } \\
\text { modules }\end{array}$ & $\begin{array}{c}\text { \# of } \\
\text { pads }\end{array}$ & $\begin{array}{c}\text { \# of } \\
\text { nets }\end{array}$ & $\begin{array}{c}\text { \# } \\
\text { pins }\end{array}$ & $\begin{array}{c}\text { \# of precedence } \\
\text { constraints }\end{array}$ \\
\hline \hline 3D-apte & 9 & 73 & 97 & 214 & 3 \\
3D-xerox & 10 & 107 & 203 & 696 & 3 \\
3D-hp & 11 & 43 & 83 & 264 & 3 \\
3D-ami33 & 33 & 42 & 123 & 480 & 7 \\
3D-ami49 & 49 & 24 & 408 & 931 & 11 \\
\hline \hline
\end{tabular}

Table 3: The five 3D-MCNC benchmark circuits.

To compare 3D-subTCG with ST, we performed two experiments. In each experiment, we set $\alpha=\beta \gamma=1$. In the first experiment, our objective is to minimize the volume with reconfiguration and communication overheads. For this experiment, we adopted the benchmark circuits used in [8] and added the reconfiguration and communication overheads. As shown in Table 2, the 3D-subTCG based method outperforms the ST-based one by a large margin. For example, 3D-subTCG achieved an average deadspace of only $19.38 \%$ while ST resulted in an average deadspace $32.01 \%$.

The second experiment is intended to test the 3D placement with the considerations of precedence constraints, wirelength, and reconfiguration/communication overheads. For this experiment, we used the MCNC benchmarks. Since the MCNC benchmarks do not have execution times and precedence constraints, we assigned their execution times and precedence constraints by ourselves. The new benchmark suite is called the 3D-MCNC

\footnotetext{
${ }^{2}$ The work [16] has been selected as one of the 40 best papers published at ICCAD during the past 20 years [13].
} 


\begin{tabular}{|c|c|c|c|c|c|c|c|c|}
\hline \multirow[t]{2}{*}{ Circuit } & \multirow[t]{2}{*}{ (\# of tasks) } & \multirow[t]{2}{*}{ Sum of Volume } & \multicolumn{3}{|c|}{ ST } & \multicolumn{3}{|c|}{ 3D-subTCG } \\
\hline & & & $\begin{array}{c}\text { Volume } \\
\left(\mathrm{mm}^{2} \times \text { clockcycles }\right)\end{array}$ & $\begin{array}{c}\text { Dead Space } \\
(\%)\end{array}$ & $\begin{array}{l}\text { Time } \\
\text { (sec.) }\end{array}$ & $\begin{array}{c}\text { Volume } \\
\left(\mathrm{mm}^{2} \times \text { colckcycles }\right)\end{array}$ & $\begin{array}{l}\text { Dead Space } \\
(\%)\end{array}$ & $\begin{array}{l}\text { Time } \\
\text { (sec.) }\end{array}$ \\
\hline beasleyl & 10 & 6218 & 8710 & 28.6 & 7.7 & (2) & 17.1 & 8.5 \\
\hline beasley2 & 17 & 11497 & 14664 & 21.5 & 45.2 & 12402 & 7.2 & 28.5 \\
\hline beasley 3 & 21 & 10362 & 16016 & 35.3 & 44.1 & 12640 & 18.0 & 22.4 \\
\hline beasley 4 & 7 & 10205 & 13800 & 26.0 & 3.0 & 13064 & 21.8 & 2.0 \\
\hline beasley5 & 14 & 16734 & 22750 & 26.4 & 18.2 & 18912 & 11.5 & 16.0 \\
\hline beasley6 & 15 & 11040 & 14994 & 26.3 & 27.9 & 13200 & 16.3 & 24.8 \\
\hline beasley7 & 8 & 17168 & 24570 & 30.1 & 3.8 & 20574 & 16.5 & 2.3 \\
\hline beasley 8 & 13 & 83044 & 132275 & 37.2 & 15.4 & 98280 & 15.5 & 19.4 \\
\hline beasley9 & 18 & 133204 & 174496 & 23.6 & 30.6 & 167751 & 20.5 & 17.2 \\
\hline beasley10 & 13 & 493746 & 660480 & 25.2 & 13.0 & 575685 & 14.2 & 10.8 \\
\hline beasley 11 & 15 & 383391 & 486381 & 24.8 & 17.5 & 438702 & 12.6 & 9.8 \\
\hline beasley 12 & 22 & 646158 & 922080 & 29.9 & 100.0 & 823816 & 21.5 & 58.5 \\
\hline okp1 & 50 & $1.24 \times 10^{8}$ & $2.16 \times 10^{8}$ & 42.6 & 1607.2 & $1.73 \times 10^{8}$ & 28.4 & 387.3 \\
\hline okp2 & 30 & $8.54 \times 10^{7}$ & $1.28 \times 10^{8}$ & 33.2 & 285.3 & $1.10 \times 10^{8}$ & 22.3 & 73.8 \\
\hline okp3 & 30 & $1.23 \times 10^{8}$ & $1.85 \times 10^{8}$ & 33.1 & 280.7 & $1.60 \times 10^{8}$ & 23.0 & 70.6 \\
\hline okp4 & 61 & $2.38 \times 10^{8}$ & $4.17 \times 10^{8}$ & 42.8 & 791.3 & $3.28 \times 10^{8}$ & 27.3 & 501.9 \\
\hline okp5 & 97 & $1.89 \times 10^{8}$ & $4.48 \times 10^{8}$ & 57.7 & 607.8 & $2.95 \times 10^{8}$ & 35.8 & 565.9 \\
\hline Average & & & & 32.01 & & & 19.38 & \\
\hline
\end{tabular}

Table 2: Results for volume optimization with reconfiguration overhead and communication overhead.

\begin{tabular}{|c|c|c|c|c|c|c|c|c|c|}
\hline \multirow[t]{2}{*}{ Circuit } & \multirow{2}{*}{$\begin{array}{l}\text { Total } \\
\text { volume }\end{array}$} & \multicolumn{4}{|c|}{ ST } & \multicolumn{4}{|c|}{ 3D-subTCG } \\
\hline & & $\begin{array}{c}\text { Volume } \\
\left(\mathrm{mm}^{2} \times \text { clockcycles }\right)\end{array}$ & $\begin{array}{l}\text { Wirelength } \\
(\mathrm{mm})\end{array}$ & $\begin{array}{c}\text { Dead space } \\
(\%)\end{array}$ & $\begin{array}{l}\text { Time } \\
\text { (sec.) }\end{array}$ & $\begin{array}{c}\text { Volume } \\
\left(\mathrm{mm}^{2} \times \text { clockcycles }\right)\end{array}$ & $\begin{array}{l}\text { Wirelength } \\
\text { (mm) }\end{array}$ & $\begin{array}{c}\text { Dead space } \\
(\%)\end{array}$ & $\begin{array}{l}\text { time } \\
\text { (sec.) }\end{array}$ \\
\hline 3D-apte & $9.88 \times 10^{7}$ & $1.18 \times 10^{8}$ & 495.0 & 16.2 & 7.7 & $1.05 \times 10^{8}$ & 335,3 & 5.9 & 3.9 \\
\hline 3D-xerox & $4.05 \times 10^{7}$ & $5.27 \times 10^{7}$ & 613.2 & 23.1 & 19.5 & $4.42 \times 10^{7}$ & 602.0 & 8.4 & 8.9 \\
\hline 3D-hp & $1.29 \times 10^{7}$ & $2.06 \times 10^{7}$ & 387.3 & 37.2 & 20.6 & $1.50 \times 10^{7}$ & 158.3 & 13.7 & 11.2 \\
\hline 3D-ami33 & $2.32 \times 10^{6}$ & $4.18 \times 10^{6}$ & 84.7 & 44.5 & 446.4 & $3.08 \times 10^{6}$ & 77.7 & 24.7 & 128.1 \\
\hline 3D-ami49 & $1.32 \times 10^{8}$ & $2.93 \times 10^{8}$ & 1040.8 & 54.9 & 1066.7 & $1.68 \times 10^{8}$ & 807.1 & 21.6 & 680.2 \\
\hline Average & & & & 35.18 & 312.18 & & & 14.86 & 166.46 \\
\hline
\end{tabular}

Table 4: Results of volume and wirelength optimization for the five 3D-MCNC benchmark circuits.

benchmark. Table 3 lists the statistics of the five 3D-MCNC benchmarks. For this experiment, we simultaneously optimized volume and wirelength with precedence constraints, and reconfiguration/communication overheads. Table 4 shows the results. As shown in Table 4, 3D-subTCG achieved better volume utilization (15\% deadspace v.s. $35 \%$ deadspace) and shorter wirelength compared to ST. 3D-subTCG also needed less CPU time than ST. Figure 12 shows the resulting placement of 3D-xerox.

Although it is hard to quantify, a key insight to the different performance between 3D-subTCG and Sequence Triplet (ST) lies in the effects of their perturbations: swapping two modules in an ST may lead to a dramatic change from the original placement while the change for the 3D-subTCG perturbation is smaller, which makes simulated annealing easier to converge to an optimal solution. (Here is an analogy: Like the gradient search for the optimization of nonlinear programming, the step size plays an important role in determining whether a search scheme can converge to the global optimal solution-a huge step size may fail to converge to an optimal solution.)

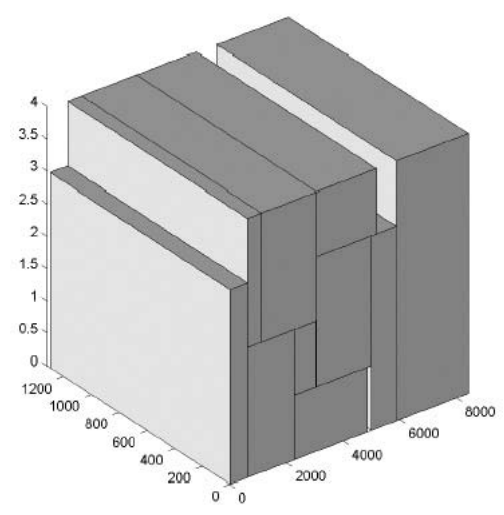

Figure 12: The result of 3D-xerox with optimizing volume and wirelength simultaneous.

\section{Conclusion}

We have presented the 3D-subTCG representation to handle the temporal floorplanning/placement problem for dynamically reconfigurable FPGAs. We have explored the feasibility conditions for the temporal relations among tasks/modules. Our algorithm can guarantee a feasible placement in each perturbation. Experimental results have shown that our method is very effective and efficient for temporal floorplanning/placement.

\section{Acknowledgements}

This work was supported in part by the National Science Council of Taiwan under Grant \# NSC 92-2213-E-002-014 and NSC 92-215-E-002-043. We also thank anonymous reviewers for their helpful comments.

\section{References}

[1] T. Cormen, C. Leiserson, and R. Rivest, Introduction to Algorithms, McGraw-Hill Book Company, 1990

[2] Atmel, "AT6000 FPGA Configuration Guide," Atmel, Inc.

[3] K. Bazargan, R. Kastner, and M. Sarrafzadeh, "Fast Template Placement for Reconfigurable Computing Systems," IEEE Design \& Test of Computers, vol.17, no. 1, pp. 68-83, Mar. 2000.

[4] K. Bazargan and M. Sarrafzadeh, "Fast Online Placement for Reconfigurable Computing Systems," IEEE Symposium on FPGAs for Custom Computing Machines, pp. 300-302, 1999.

[5] K. Bazargan, R. Kastner and M. Sarrafzadeh, "3-D Floorplanning: Simulated Annealing and Greedy Placement Methods for Reconfigurable Computing Systems," Design Automation for Embedded Systems - RSP'99 Special Issue, Apr. 2000.

[6] J. E. Beasley, "An Exact Two-Dimensional Non-Guillotine Cutting Stock Tree Search Procedure," Operations Research, vol.33, no. 1, pp. 49-64, 1985.

[7] S. P. Fekete, E. Köhler, and J. Teich, "Optimal FPGA Module Placement with Temporal Precedence Constraints," Proc. DATE, pp. 658-665, Mar. 2001.

[8] S. P. Fekete, and J. Schepers, "On more-dimensional packing III: Exact Algorithms," ZPR Technical Report 97-290 1997.

[9] M. Gokhale, B. Holmes, A. Kopster, D. Kunze, D. Lopresti, S. Lucas, R. Minnich, and P. Olsen, "Splash: A Reconfigurable Linear Logic Array," International Conference on Parallel Processing, pp. 526-532, 1990.

[10] S. Hauck, "The Roles of FPGAs in Reprogrammable Systems," Proc. of the IEEE, vol.86, no. 4, pp. 615-639, Apr. 1998.

[11] S. Hauck, Z. Li, and E.J. Schwabe, "Configuration Compression for the Xilinx XC6200 FPGA," Proc. of the IEEE Symposium on FPGAs for Custom Computing Machines, pp. 138-146, 1998

[12] S. Kirkpatrick, C. D. Gelatt, and M. P. Vecchi, "Optimization by Simulated Annealing," Science, vol. 220, no. 4598, pp.671-680, May, 1983.

[13] A. Kuehlmann, Ed., The Best of ICCAD-20 Years of Excellence in Computer-Aided Design, Kluwer Academic Pub., 2003.

[14] E. Lawler, Combinatorial Optimization: Networks and Matroids, Holt, Rinehart, and Winston, 1976.

[15] J.-M. Lin and Y.-W Chang, "TCG: A Transitive Closure Graph-Based Representation for Non-Slicing Floorplans," Proc. DAC, pp. 764-769, June 2001.

[16] H. Murata, K. Fujiyoshi, S. Nakatake, and Y. Kajitani, "Rectangle-Packing Based Module Placement," Proc. ICCAD, pp. 472-479, 1995.

[17] J. Teich, S. P. Fekete, and J. Schepers, "Compile-Time Optimization of Dynamic Hardware Reconfigurations," Proc PDPTA, pp. 1097-1103, June 1999.

[18] S. Trimberger, "A Time-Multiplexed FPGA," Proc. FCCM'97.

[19] Xilinx, "XC6200 Field Programmable Gate Arrays Data Sheet," Xilinx, Inc., Oct. 1996.

[20] Xilinx, "XAPP151 Virtex Series Configuration Architecture User Guide v1.5," Xilinx, Inc., Sep. 2000. 